\title{
Mechanism of Interaction Between Cefepime and Papain by Spectroscopic Method
}

\author{
Li Chen, Baosheng Liu*, Chundan Wang, Lihua Ma, Hongcai Zhang, Xu Guo \\ College of Chemistry \& Environmental Science, Hebei University, Baoding, P. R. China \\ Email address: \\ lbs@hbu.edu.cn (Baosheng Liu) \\ ${ }^{*}$ Corresponding author
}

To cite this article:

Li Chen, Baosheng Liu, Chundan Wang, Lihua Ma, Hongcai Zhang, Xu Guo. Mechanism of Interaction Between Cefepime and Papain by Spectroscopic Method. Journal of Photonic Materials and Technology. Vol. 4, No. 1, 2018, pp. 33-38. doi: 10.11648/j.jmpt.20180401.16

Received: January 27, 2018; Accepted: February 16, 2018; Published: March 19, 2018

\begin{abstract}
The reaction mechanism of cefepime (CEF) with papain (PAPA) was investigated by both fluorescence quenching and synchronous fluorescence spectroscopy in different temperature $(293,308$ and $318 \mathrm{~K})$. The results demonstrated that the interaction between CEF and PAPA was taking place via static quenching. Thermodynamic parameters revealed that electrostatic interaction played major roles in the interaction. The order of magnitudes of binding constant is $10^{4}$, and the number of binding site in the system was closed to 1 . The binding rates of CEF to PAPA $\left(\lambda_{e x}=280 \mathrm{~nm}\right)$ were $81.46 \% \sim 95.95 \%(293 \mathrm{~K})$, $80.50 \% \sim 95.49 \%(308 \mathrm{~K})$ and $79.77 \% \sim 95.12 \%(318 \mathrm{~K})$. There was a linear relationship between $\Delta F$ and the concentrations of $\mathrm{CEF}$ in the range of $6.0 \times 10^{-6} \sim 7.0 \times 10^{-5} \mathrm{~mol} / \mathrm{L}$. And the correlation coefficient of the linear curve was 0.9973 .
\end{abstract}

Keywords: Cefepime, Papain, Reaction Mechanism, Spectroscopy, Determination

\section{Introduction}

Cefepime (CEF) is a fourth-generation cephalosporin with antibacterial spectrum covering aerobic gram-positive and gram-negative bacteria including Pseudomonas aeruginosa [1]. CEF has been recommended for a wide array of infections such as hospitalacquired pneumonia, febrile neutropenia, soft tissue infections and intra-abdominal infections [2]. The structure of CEF was shown in figure 1.

Papain (PAPA), a proteolytic enzyme from carica papaya with a molecular mass of 23, $406 \mathrm{Da}$ [3]. PAPA was first described in 1879 (Wurtz \& Bouchut, 1879), a large database of information on the enzyme has been gathered [4]. PAPA fruits and leaves are traditionally used in South-East Asia and Africa for wound healing and treatment of inflammation (e.g., during burns). PAPA fruits contain considerable amounts of proteolytic enzymes that promote wound sanation from necrotized tissue [5]. The study of the interaction between proteins and endogenous compounds as well as many drug molecules has been attracting the attention of people. Studing the binding mechanism of protein-ligands has very important significance for the life sciences, chemistry, pharmacology and clinical medicine [6]. The interaction between PAPA and various drugs has been reported rarely.<smiles>CO/N=C(/C(=O)NC1C(=O)N2C(C(=O)[O-])=C(C[N+]3(C)CCCC3)CSC12)c1csc(N)n1</smiles>

Figure 1. Chemical structure of cefepime. 


\section{Experimental}

\subsection{Apparatus}

All fluorescence spectra were recorded with a Shimadzu RF-5301PC spectrofluorophotometer. All pH measurements were carried out with a pHS-3C precision acidity meter (Leici, Shanghai, China). All temperatures were controlled by a SYC-15 B superheated water bath (Nanjing Sangli Electronic Equipment Factory).

\subsection{Materials}

PAPA was purchased from Sigma-Aldrich (purity grade inferior 99\%, Shanghai, China). Stock solutions of CEF standard substance (CAS\#, 29094-61-9)(5.0 $\left.\times 10^{-5} \mathrm{~mol} / \mathrm{L}\right)$ and PAPA $\left(4.0 \times 10^{-4} \mathrm{~mol} / \mathrm{L}\right)$ were prepared. All the stock solutions were further diluted for use as working solutions. Tris-HCl buffer solution containing $0.15 \mathrm{~mol} / \mathrm{L} \mathrm{NaCl}$ was used to maintain the $\mathrm{pH}$ of solutions at 7.40 and $\mathrm{NaCl}$ solution was used to maintain the ionic strength of the solution. All other reagents were of analytical grade and all aqueous solutions were prepared with newly double-distilled water and stored in dark at $277 \mathrm{~K}$.

The fluorescence intensities were corrected for the absorption of excitation light and re-absorption of emitted light to decrease the inner filter using the following relationship [7].

$$
F_{c o r}=F_{o b s} \times e^{\left(A_{e x}+A_{e m}\right) / 2}
$$

Where, $F_{\text {cor }}$ and $F_{\text {obs }}$ are the corrected and observed fluorescence intensities, respectively. $A_{\mathrm{ex}}$ and $A_{\mathrm{em}}$ are the absorbance values of CFS at excitation and emission wavelengths, respectively. The fluorescence intensity used in this paper was corrected.

\subsection{Procedures}

\subsubsection{Fluorescence Measurements}

In a typical fluorescence measurement, $1.0 \mathrm{~mL}$ Tris- $\mathrm{HCl}$ ( $\mathrm{pH}=7.40), 1.0 \mathrm{~mL}$ PAPA solution $\left(6.0 \times 10^{-6} \mathrm{~mol} / \mathrm{L}\right)$, and different concentrations of CEF were added into $10 \mathrm{~mL}$ colorimetric tubes successively. The samples were diluted to $10.0 \mathrm{~mL}$ with double-distilled water, mixed thoroughly by shaking, and kept static for $30 \mathrm{~min}$ at different temperatures (293, 308 and $318 \mathrm{~K})$. The excitation wavelength for PAPA was $280 \mathrm{~nm}$ and $295 \mathrm{~nm}$, respectively, with a $1.0 \mathrm{~cm}$ quartz sampling cells. The excitation and emission slits were set at 5 $\mathrm{nm}$. The solution was subsequently scanned on the fluorophotometer and the fluorescent intensity of the system was recorded.

\subsubsection{Synchronous Fluorescence Measurements}

Solution was prepared as above. The fluorescence spectra of the CEF-PAPA systems were recorded when the $\Delta \lambda$ value between the excitation and emission wavelengths was stabilized at 15 and $60 \mathrm{~nm}$, respectively.

\section{Results and Discussion}

\subsection{Fluorescence Spectra of CEF-PAPA Systems}

Thus far, fluorescence spectroscopy has been regarded as the most comprehensive method for studying protein-ligand interactions. As a result, we employed fluorescence spectroscopy to investigate the interaction between CEF and PAPA. The fluorescence spectra of PAPA with different concentrations of CEF were determined and have been shown in figure 2.

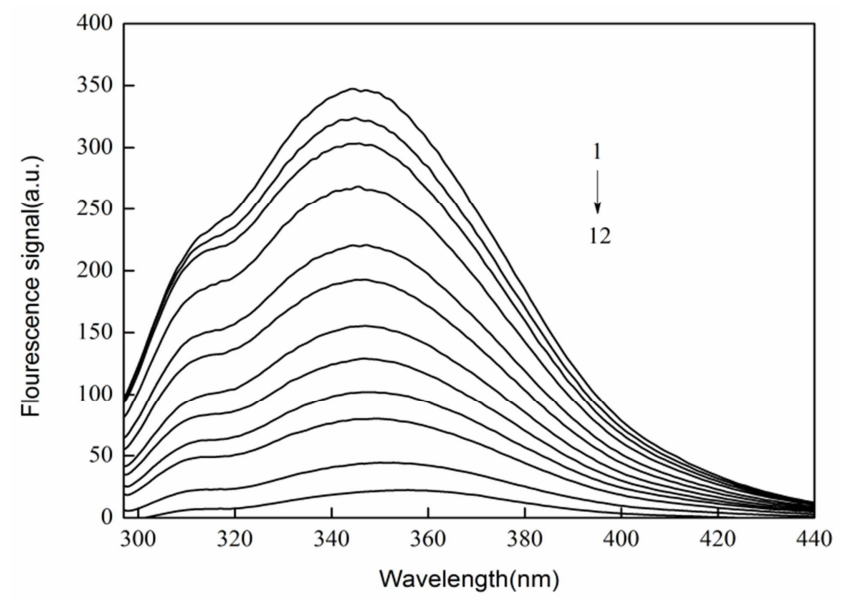

Figure 2. Fluorescence emission spectra of CEF-PAPA ( $T=293 \mathrm{~K}, \lambda_{e x}=280$ nm) $C_{P A P A}=6.0 \times 10^{-6} \mathrm{~mol} / \mathrm{L}, 1 \sim 10: C_{C E F}=(0,0.4,0.6,0.6,1.0,2.0,3.0,4.0,5.0$, 6.0, $8.09 .0,10,16,20) \times 10^{-5} \mathrm{~mol} / \mathrm{L}$.

PAPA fluorescence intensity decreased remarkably as CEF concentrations increased. In general, the molecular interactions such as energy transfer, ground-state complex formation, molecular rearrangements, and collision quenching can contribute to fluorescence quenching. There are two possible modes for fluorescence quenching: static quenching and dynamic quenching. To determine the quenching mechanism of PAPA by CEF, the fluorescence quenching data were analyzed in terms of the Stern-Volmer equation:

$$
I_{0} / I=1+K_{q} \tau_{0}[L]=1+K_{s v}[L]
$$

where $I_{0}$ and $I$ denote the fluorescence intensities of PAPA in the absence and presence of CEF, respectively. $K_{s v}$ is the Stern-Volmer dynamic quenching constant, which can be determined by linear regression of a plot of $I_{0} / I$ against $[L]$. $K q$ represents the quenching rate constant of the bimolecular and the value of the maximum scattering collision quenching constant is $2.0 \times 10^{10} \mathrm{~mol} \cdot \mathrm{L}^{-1} \cdot \mathrm{S}^{-1}$ and $\tau_{0}$ denotes the lifetime of the fluorophore without a quencher $\left(\tau_{0} \approx 1 \times 10^{-8} \mathrm{~s}\right)$ [8].

The $K_{s v}$ and $K_{q}$ values derived from Eq. (2) at the three temperatures have been presented in Table 1. All the values of $K q$ were much greater than that of the maximum scattering collision quenching constant $\left(2.0 \times 10^{10} \mathrm{~mol} \cdot \mathrm{L}^{-1} \cdot \mathrm{S}^{-1}\right)$, indicating that the fluorescence quenching mechanism of PAPA initiated by $\mathrm{CEF}$ was a static quenching procedure. In addition, It could 
be observed that $K_{s v}$ and $K_{q}$ values were inversely correlated with temperature. It could thus be assumed that the quenching mechanism was due to complex formation between CEF and PAPA, rather than dynamic collision.

For static quenching process, the binding constants $\left(K_{a}\right)$ and the number of binding sites $(n)$ can be calculated using the following Eq. (3)[9].

$$
\lg \left(\frac{I_{0}-I}{I}\right)=n \lg K_{a}+\mathrm{n} \lg \left\{[L]-n \frac{I_{0}-I}{I_{0}}\left[B_{t}\right]\right\}
$$

where $[L]$ and $\left[B_{t}\right]$ are the total concentrations of CEF and PAPA, respectively. On the assumption that $n$ in the bracket is equal to 1 , the curve of $\lg \left(I_{0}-I\right) / I$ versus $\lg \left\{[L]-\left[B_{t}\right]\left(F_{0}-F\right) / F_{0}\right\}$ is drawn and fitted linearly, then the value of $n$ can be obtained from the slope of the plot. If the $n$ value obtained is not equal to 1 , then it is substituted into the bracket and the curve of $l g$ $\left(I_{0}-I\right) / I$ versus $\lg \left\{[L]-\left[B_{t}\right]\left(I_{0}-I\right) / I_{0}\right\}$ is drawn again. This process is repeated again and again till a single value is obtained for $n$. On the basis of the value of $n$ obtained, the binding constant $K_{a}$ can also be determined [10]. The results are listed in Table 1. From the results, all the values of $n$ were approximately equal to 1 at different temperatures, implying that there was just one binding site for CEF existed in PAPA. Meanwhile, the binding constants $K_{a}$ decreased with increasing temperature, which implied the existence of CEF-PAPA complex and their stability became less stable with rising temperature and the quenching was a static process.

Table 1. Quenching reactive parameters of CEF-PAPA system at different temperatures.

\begin{tabular}{llllllll}
\hline$\lambda_{\text {ex }}(\mathbf{n m})$ & $\boldsymbol{T} /(\boldsymbol{K})$ & $\boldsymbol{K}_{q} /(\mathbf{L} / \mathbf{m o l} \cdot \mathbf{s})$ & $\boldsymbol{K}_{\boldsymbol{s v}} /(\mathbf{L} / \mathbf{m o l})$ & $\boldsymbol{r}_{\boldsymbol{l}}$ & $\boldsymbol{K}_{\boldsymbol{a}} /(\mathbf{L} / \mathbf{m o l})$ & $\boldsymbol{n}$ & $\boldsymbol{r}_{\mathbf{2}}$ \\
$\lambda_{\text {ex }}=280 \mathrm{~nm}$ & 293 & $2.61 \times 10^{12}$ & $2.61 \times 10^{4}$ & 0.9958 & $2.37 \times 10^{4}$ & 1.24 & 0.9956 \\
& 308 & $2.32 \times 10^{12}$ & $2.32 \times 10^{4}$ & 0.9996 & $2.12 \times 10^{4}$ & 1.08 & 0.9983 \\
& 318 & $1.93 \times 10^{12}$ & $1.93 \times 10^{4}$ & 0.9971 & $1.95 \times 10^{4}$ & 0.96 & 0.9963 \\
$\lambda_{\text {ex }}=295 \mathrm{~nm}$ & 293 & $2.06 \times 10^{12}$ & $2.06 \times 10^{4}$ & 0.9990 & $1.96 \times 10^{4}$ & 1.13 & 0.9996 \\
& 308 & $1.56 \times 10^{12}$ & $1.56 \times 10^{4}$ & 0.9999 & $1.82 \times 10^{4}$ & 1.22 & 0.9984 \\
& 318 & $1.33 \times 10^{12}$ & $1.33 \times 10^{4}$ & 0.9994 & $1.49 \times 10^{4}$ & 1.06 & 0.9996 \\
\hline
\end{tabular}

$r_{l}$ is the linear relative coefficient of $I_{0} / I \sim[L] ; r_{2}$ is the linear relative coefficient of $\lg \left(I_{0}-I\right) / I \sim \lg \left\{[L]-n\left[B_{t}\right]\left(I_{0}-I\right) / I_{0}\right\}$.

\subsection{Synchronous Fluorescence Spectra of CEF-PAPA Systems}

Synchronous fluorescence spectroscopy is a simple and sensitive technique to measure the probable shift of the maximum emission wavelength, and probe the micro-environmental changes of the chromophores. In order to investigate the structural change of PAPA in the presence of CEF, we measured synchronous fluorescence spectra of PAPA. In this method, $\Delta \lambda$ stands for the value of the difference between excitation and emission wavelengths. When is $15 \mathrm{~nm}$ or $60 \mathrm{~nm}$, the synchronous fluorescence spectroscopy just shows the spectroscopic behavior of tyrosine (Tyr) residue or tryptophan (Trp) residue of proteins, separately. It is generally known that when the peak position moves to the long wave direction, the exposure extent of the residue is increased, and the microenvironment of the residues would be more hydrophilic. However, when the peak position moves to the short wave direction, the consequence is opposite [11].

To explore the structural change of the PAPA by addition of CEF, we recorded the synchronous fluorescence spectra of the PAPA at various concentrations of CEF. It can be seen from figure 3 that the fluorescence intensity of both Tyr and Trp residues decreased regularly, but the peak position of the Tyr residues moves to the long wave length direction with increasing concentration of CEF, indicated that the polarity around the tyrosine residues was increased. and that the hydrophobicity was decreased. At the same time, there was no change in the peak positionof Trp residues, indicating that the polarity around the Trp residues has not changed.
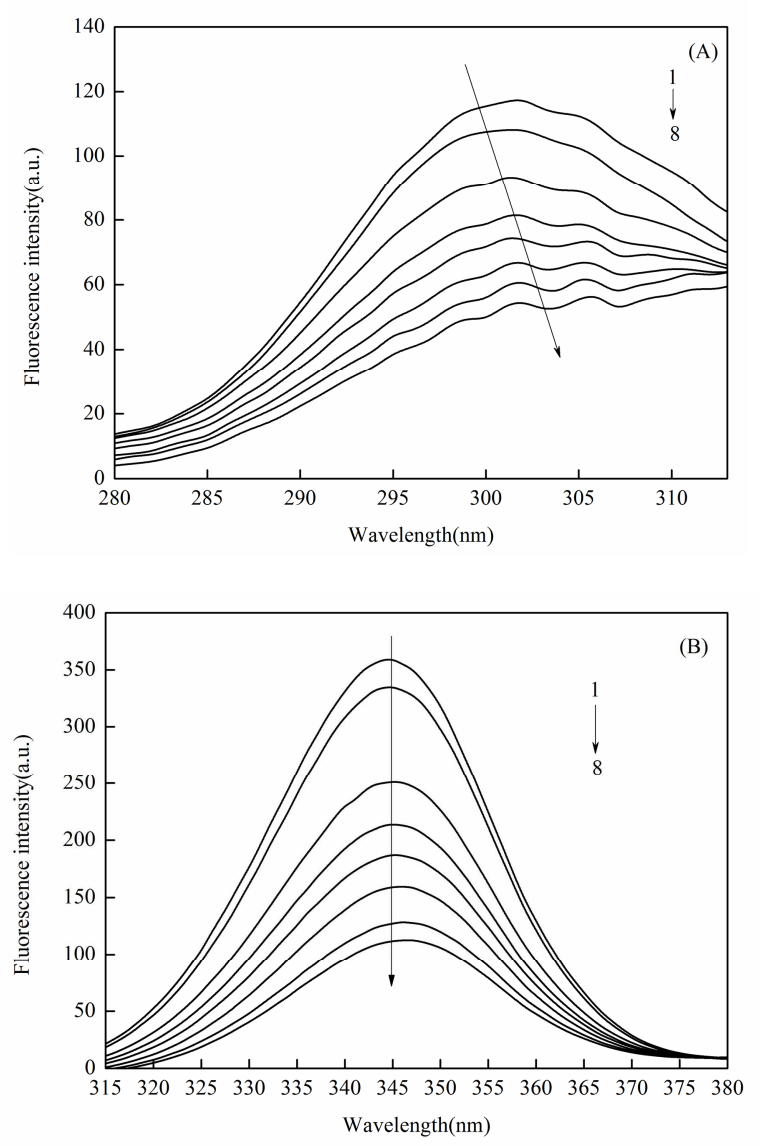

Figure 3. Fluorescence spectrum of CEF-PAPA system $(T=293 \mathrm{~K})$ (A) $\Delta \lambda=15 \mathrm{~nm} ;(B) \Delta \lambda=60 \mathrm{~nm} C_{P A P A}=6.0 \times 10^{-6} \mathrm{~mol} / \mathrm{L}, 1 \sim 8: C_{C E F}=(0,0.6,2.0,3.0$, 5.0, 6.0, 7.0, 8.0) $\times 10^{-5} \mathrm{~mol} / \mathrm{L}$. 


\subsection{The Participation of Amino Acid Residue Studies in CEF-PAPA Systems}

In order to infer the participation and reaction degree of Trp and Tyr residues in CEF-PAPA system, fluorescence and synchronous fluorescence experiments were performed, as shown in figure 4 . In figure 4 (A), when $n_{C E F}: n_{P A P A}<7: 1$, the quenching curves of PAPA excited at $280 \mathrm{~nm}$ and $295 \mathrm{~nm}$ approximately overlap, and only the Trp residues participate in the reaction. When $n_{C E F}: n_{P A P A}>7: 1$, and the extent of fluorescence quenching at $280 \mathrm{~nm}$ was larger than the extent of fluorescence quenching at $295 \mathrm{~nm}$. This showed that Trp and Tyr residues both participated in the CEF-PAPA complexation process. However, in figure 4 (B), Trp was involved in the whole reaction, but the extent of the reaction is not as large as that of Tyr. In addition, from Table 1, at the same temperature, the binding constant $K_{a}\left(\lambda_{e x}=280 \mathrm{~nm}\right)$ was obviously greater than the binding constant $K_{a}\left(\lambda_{e x}=295 \mathrm{~nm}\right)$; the binding constant $K_{a}(\Delta \lambda=60 \mathrm{~nm})$ was obviously greater than the binding constant $K_{a}(\Delta \lambda=15 \mathrm{~nm})$.

Synchronous fluorescence quenching ratio, denoted by $R_{(\Delta \lambda)}$, represents the percentage of decrease in fluorescence intensity. The larger the quenching ratio was, the greater the degree of quenching was.

$$
R(\Delta \lambda)=1-I / I_{0}
$$

where $I_{0}$ and $I$ denote the fluorescence intensities of PAPA in the absence and presence of CEF, respectively.

The values of $R_{(\Delta \lambda=15 \mathrm{~nm})}$ and $R_{(\Delta \lambda=60 \mathrm{~nm})}$ were calculated according to Eq. (4). The ratio of Tyr residue in CEF-PAPA system was $40.13 \%(293 \mathrm{~K}), 46.12 \%(308 \mathrm{~K})$, and $49.91 \%$ (318K), and the ratio of Tyr residue in CEF-PAPA system was calculated by $R_{(\Delta \lambda=15 \mathrm{~nm})} /\left(R_{(\Delta \lambda=15 \mathrm{~nm})}+R_{(\Delta \lambda=60 \mathrm{~nm})}\right)$. From the data, it can be seen that the binding ratio of Tyr residue increases with increasing temperature. The ratio of Trp residue in CEF-PAPA system was $59.86 \%$ (293K), 53.87\% (308K), and $50.09 \%$ (318K), and the ratio of Trp residue was calculated by $R_{(\Delta \lambda=60 \mathrm{~nm})} /\left(R_{(\Delta \lambda=15 \mathrm{~nm})}+R_{(\Delta \lambda=60 \mathrm{~nm})}\right)$. It is obvious that the binding ratio of Trp decreases with increasing temperature. It can be concluded that Trp and Tyr residues both were involved in the reaction of the CEF-PAPA system, and Trp residues contributed more than Tyr residues in the reaction.

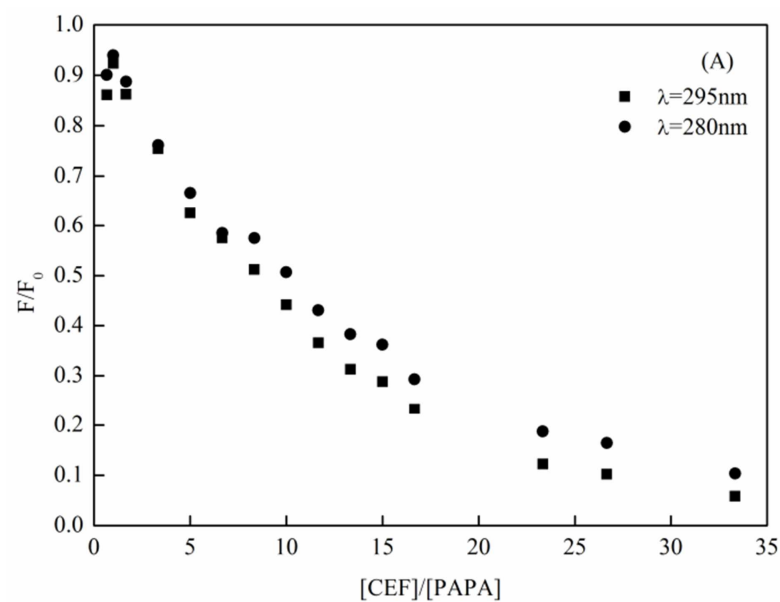

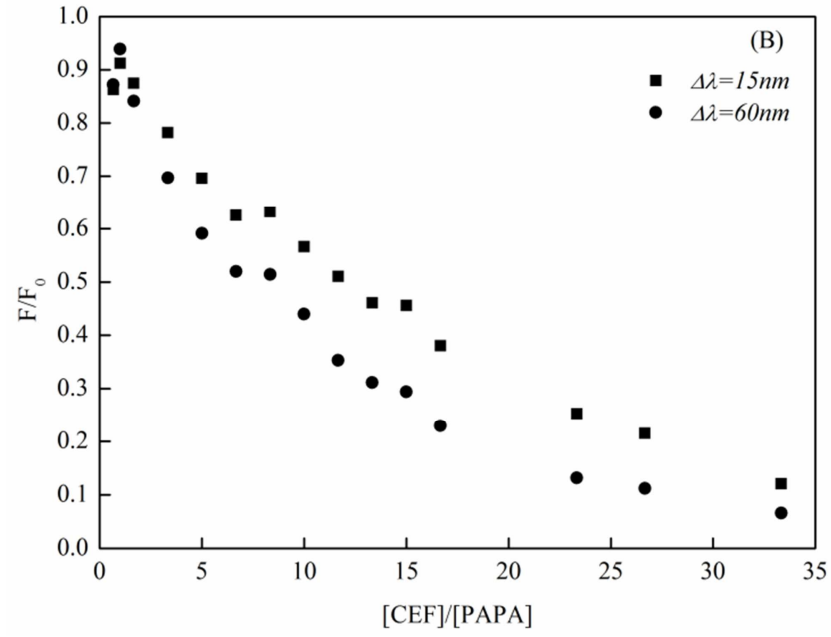

Figure 4. Quenching curves of CEF-PAPA system at $\lambda_{e x}=280 \mathrm{~nm}, \lambda_{e x}=295 \mathrm{~nm}$, $\Delta \lambda=15 \mathrm{~nm}$ and $\Delta \lambda=60 \mathrm{~nm}(T=293 \mathrm{~K}) C_{P A P A}=6.0 \times 10^{-6} \mathrm{~mol} / \mathrm{L}, 1 \sim 10: C_{C E F}=(0$, 0.4, 0.6, 1.0, 2.0, 3.0, 4.0, 5.0, 6.0, 7.0, 8.0, 9.0, 10, 14, 16, 20) $\times 10^{-5} \mathrm{~mol} / \mathrm{L}$.

\subsection{Type of Interaction Force of CEF-PAPA Systems}

The interaction forces between a small molecule and macromolecule include hydrogen bonds, van der Waals force, hydrophobic force, electrostatic interactions, etc. The thermodynamic parameters of binding reaction are the main evidence for confirming the binding force [12].

The enthalpy change $(\Delta H)$ and entropy change $(\Delta S)$ can be evaluated from the Van't Hoff equation

$$
R \ln K=\Delta S-\Delta H / T
$$

Where $K$ is binding constant at the corresponding temperature, $T$ is the experimental temperature and $R$ is the gas constant.

To expound the interaction between CEF and PAPA, the thermodynamic parameters were calculated using equations (5) and (6) by the linear curve fitting method (figure 5) and are given in Table 2 .

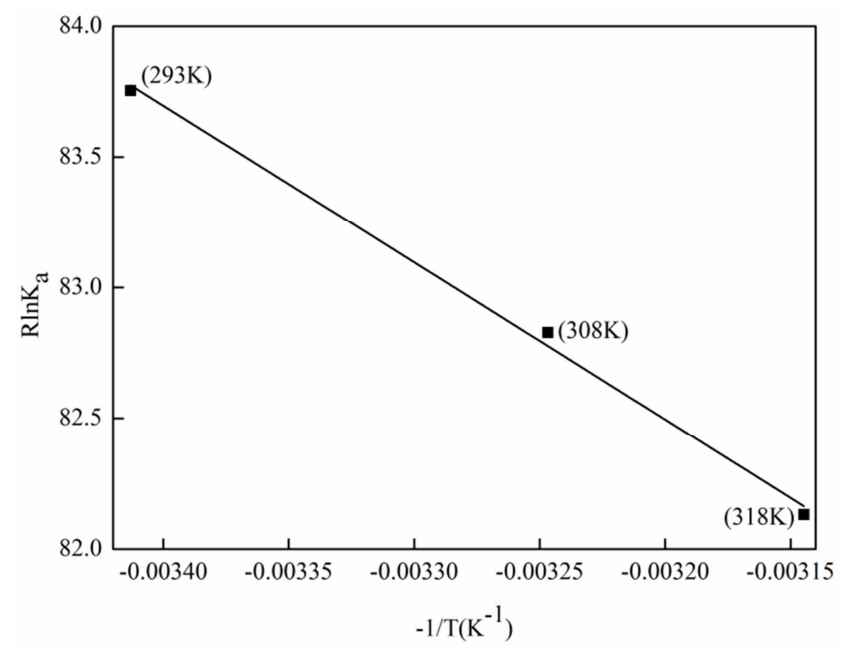

Figure 5. Vant s-Hoff plot for the interaction of CEF and PAPA at different temperatures.

From figure 5, we can see the good linear relationship 
between $\ln K$ and $1 / T(r=0.9985)$. The free energy change $(\Delta G)$ was then estimated from the following relationship.

$$
\Delta G=\Delta H-T \Delta S
$$

The calculated thermodynamic parameters $\Delta H, \Delta S$ and $\Delta G$ are also listed in Table 2. From Table 2, the negative values of $\triangle G$ revealed that the interaction between CEF and PAPA is spontaneous. Both $\Delta H$ is negative value but $\Delta S$ is positive value, which can be concluded that the acting forces during the interaction between CEF and PAPA are mainly electrostatic interactions.

Table 2. The thermodynamic parameters of CEF-PAPA system at different temperatures $\left(\lambda_{\text {ex }}=280 \mathrm{~nm}\right)$.

\begin{tabular}{|c|c|c|c|c|}
\hline$T /(\mathbf{K})$ & $\mathrm{K}_{a} /\left(\mathrm{L} \cdot \mathrm{mol}^{-1}\right)$ & $\Delta H /\left(\mathrm{KJ} \cdot \mathrm{mol}^{-1}\right)$ & $\Delta S /\left(\mathrm{J} \cdot \mathrm{mol}^{-1} \cdot \mathrm{K}^{-1}\right)$ & $\Delta G /\left(\mathrm{KJ} \cdot \mathrm{mol}^{-1}\right)$ \\
\hline 293 & $2.37 \times 10^{4}$ & & 63.28 & -24.54 \\
\hline 308 & $2.12 \times 10^{4}$ & -5.999 & 63.35 & -25.51 \\
\hline 318 & $1.95 \times 10^{4}$ & & 63.27 & -26.11 \\
\hline
\end{tabular}

\subsection{Binding rate of CEF to PAPA}

The binding constants and number of binding sites are combined to establish a theory model to predict the binding rate of drug to plasma protein. When the number of binding sites is equal to 1 , the binding rate of CEF to PAPA $(W)$ can be obtained from the following equations [13]:

$$
W=\frac{\left(K_{a} R+K_{a}+\frac{1}{\left[B_{0}\right]}\right)-\sqrt{\left(K_{a} R+K_{a}+\frac{1}{\left[B_{0}\right]}\right)^{2}-4 K_{a}^{2} R}}{2 K_{a} R}
$$

$$
K_{a} R\left[B_{0}\right] W^{2}-\left(K_{a} R\left[B_{0}\right]+K_{a}\left[B_{0}\right]+1\right) W+K_{a}\left[B_{0}\right]=0
$$

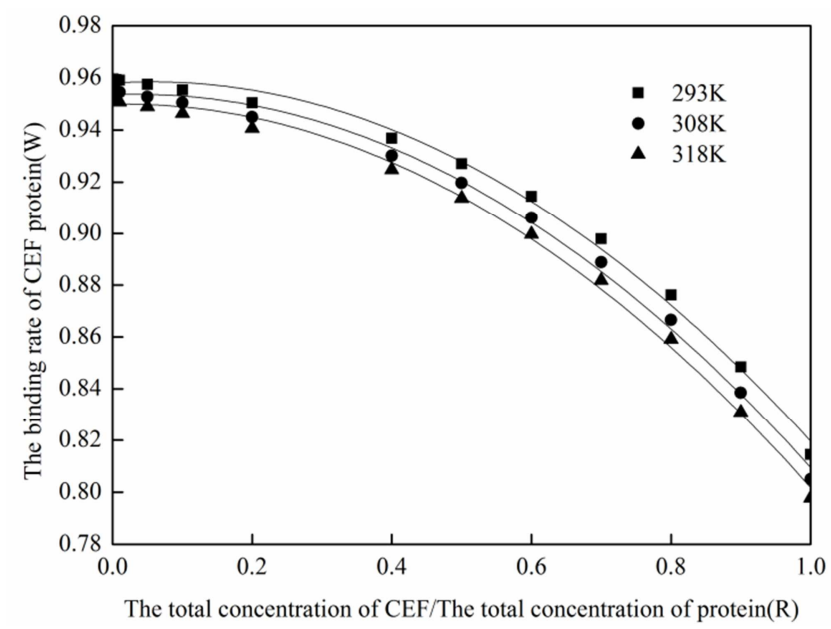

Figure 6. The binding rate of CEF to protein in different temperature $\left(\lambda_{e x}=280 \mathrm{~nm}\right)$.

Where $K_{a}$ is the binding constant; $\left[B_{0}\right]$ is the total concentration of protein; $\left[L_{0}\right]$ is the total concentration of the drug; $R$ is the ratio of the total concentration of the drug with protein $\left(\left[L_{0}\right] /\left[B_{0}\right]\right)$. In order to reflect the actual significance of the experiment, the content in the PAPA of approximately $1.0 \times 10^{-3} \mathrm{~mol} / \mathrm{L}$ is chosen. The binding rate of $\mathrm{CEF}$ $\left(1.0 \times 10^{-7} \sim 1.0 \times 10^{-3} \mathrm{~mol} / \mathrm{L}\right)$ to PAPA is then calculated using Eq. (9) at three temperatures. Figure 6 shows plots of W versus R according to the Eq. (10). Using the $K_{a}$ values and Eq. (9), the binding rates of different CEF concentrations to PAPA at $293,308,318 \mathrm{~K}$ were calculated $\left(\lambda_{e x}=280 \mathrm{~nm}\right)$, respectively. That is: $\quad 81.46 \% \sim 95.95 \%, \quad 80.50 \% \sim 95.49 \%$, and
$79.77 \% \sim 95.12 \%$. The curve of $293 \mathrm{~K}$ deal with nonlinear fitting, obtaining the equation: $y=-0.15357 x^{2}+0.00547 x+0.94988, \quad r=0.9973, \quad$ that is $W=-0.15357 R^{2}+0.00547 R+0.94988, r=0.9973$. From Figure 6 and the fitting equation, a decrease in the binding rate $W$ is observed with an increase in concentration of CEF at a fixed PAPA concentration. The protein binding rate of this drug provides an important reference value for reasonable clinical medication.

\subsection{Standard Curve in CEF-PAPA Systems}

According to the above experimental method, different concentrations of CEF were added into PAPA solution at 318 $\mathrm{K}$ to investigate the relationship between the fluorescence intensity $\Delta F\left(\Delta F=F_{0}-F\right)$ and the CEF concentration. As shown in figure 7 . There was a linear relationship between $\Delta F$ and the concentrations of $\mathrm{CEF}$ in the range of $6.0 \times 10^{-6} \sim 7.0 \times 10^{-5} \mathrm{~mol} / \mathrm{L}$ with the correlation coefficient of 0.9973. The linear equation could be described as $\Delta F=2.21 \times 10^{6} \mathrm{C}(\mathrm{mol} / \mathrm{L})+20.10$.

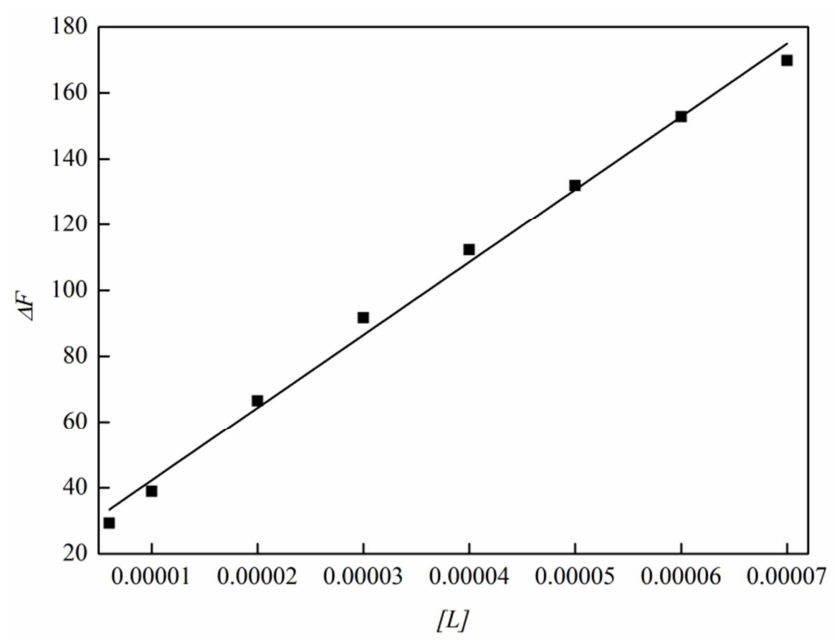

Figure 7. The standard curve in CEF-PAPA systems at $318 \mathrm{~K}\left(\lambda_{e x}=280 \mathrm{~nm}\right)$.

\section{Conclusions}

In this article, the interaction between CEF and PAPA was studied at different temperatures by two different fluorescence spectrometries. The result showed that $\mathrm{CEF}$ quenched the fluorescence of PAPA by static quenching. Electrostatic 
interaction was the main driving power in the binding process. In PAPA molecules, Tyr and Trp residues both involved in reaction. Synchronous fluorescence spectrometry has some advantages such as good selectivity, high sensitivity and less interference, which makes it more useful than fluorescence quenching method in investigating the interaction mechanism between drugs and proteins. At the same time, the performed results provide some important information on the structure and function of protein and may be useful for pharmacology.

\section{Acknowledgements}

The authors gratefully acknowledge financial support of the National Science Foundation of China (Grant no. 20675024) and the Hebei Provincial Key Basic Research Program (Grant No. 10967126D).

\section{References}

[1] S. V. Burgess, V. H. Mabasa, I. Chow, M. H. Ensom, J. Annals of Pharmacotherapy, 2015, 49, 3.

[2] A. Meillier, D. Rahimian, J. Oxford Medical Case Reports, 2016, 2016, 6 .

[3] J. Yao, C. Lin, T. Tao, F. Lin, J. Colloids \& Surfaces B Biointerfaces, 2013, 101, 101C.
[4] R. Menard, H. E. Khouri, C. Plouffe, R. Dupras, D. Ripoll, T. Vernet, D. C. Tessier, F. Laliberte, D. Y. Thomas, A. C. Storer, J. Biochemistry, 1990. 29, 28.

[5] E. V. Mikhalchik, A. V. Ivanova, M. V. Anurov, S. M. Titkova, L. Y. Penkov, Z. F. Kharaeva, L. G. Korkina, J. Bulletin of Experimental Biology \& Medicine, 2004, 137 (6):560-562.

[6] A. B. Khan, J. M. Khan, M. S. Ali, R. H. Khan, Kabir-ud-Din, J. Spectrochimica Acta Part A Molecular \& Biomolecular Spectroscopy, 2012, 97, 6.

[7] Q. Wang, J. He, J. Yan, D. Wu, H. Li, J. Luminescence, 2015, 30,2 .

[8] Y. Liu, G. Zhang, Y. Liao, Y. Wang, J. Spectrochimica Acta Part A Molecular \& Biomolecular Spectroscopy, 2015, 151, 498.

[9] L. Trnková, I. Boušová, V. Staňková, J. Dršata, J. Journal of Molecular Structure, 2011, 985, 2.

[10] R. Han, B. Liu, G. Li, Q. Zhang, J. Spectroscopy Letters, 2016, 49,3 .

[11] G. Wang, Y. Chen, C. Yan, Y. Lu, J. Journal of Luminescence, 2015, 157, 229.

[12] G. Zhang, X. Chen, J. Guo, J. Wang, J. Journal of Molecular Structure, 2009, 921, 1-3.

[13] Q. J. Zhang, B. S. Liu, R. Han, G. X. Li, J. Spectroscopy Letters, $2015,49,3$. 